\title{
Microstructural evaluation of primary crystallization with diffusion-controlled grain growth
}

\author{
D. Crespo* \\ Departament de Física Aplicada, Universitat Politècnica de Catalunya, Campus Nord UPC, Mòdul B4, O8034 Barcelona, Spain \\ T. Pradell \\ Escola Universitària d'Enginyeria Tècnica Agricola, Universitat Politècnica de Catalunya, Urgell 187, 08036 Barcelona, Spain \\ M. T. Clavaguera-Mora \\ Grup de Física de Materials I, Departament de Física, Facultat de Ciències, Universitat Autònoma de Barcelona, \\ 08193 Bellaterra, Spain \\ N. Clavaguera \\ Grup de Física de l'Estat Sòlid, Departament d'ECM, Facultat de Física, Universitat de Barcelona, Diagonal, 647, \\ 08028 Barcelona, Spain \\ (Received 3 July 1996)
}

\begin{abstract}
A model has been developed for evaluating grain size distributions in primary crystallizations where the grain growth is diffusion controlled. The body of the model is grounded in a recently presented mean-field integration of the nucleation and growth kinetic equations, modified conveniently in order to take into account a radius-dependent growth rate, as occurs in diffusion-controlled growth. The classical diffusion theory is considered, and a modification of this is proposed to take into account interference of the diffusion profiles between neighbor grains. The potentiality of the mean-field model to give detailed information on the grain size distribution and transformed volume fraction for transformations driven by nucleation and either interfaceor diffusion-controlled growth processes is demonstrated. The model is evaluated for the primary crystallization of an amorphous alloy, giving an excellent agreement with experimental data. Grain size distributions are computed, and their properties are discussed. [S0163-1829(97)10405-2]
\end{abstract}

\section{INTRODUCTION}

For some nonequilibrium transformations the kinetics of molecular rearrangement is sufficiently slow to give a rate of advance of the interface limited by the kinetics of the interface attachment process rather than by the heat flow. This characteristic has been used to carry out studies of interfacecontrolled crystal growth over wide ranges of temperature. We wish to consider the situation in which the kinetics of transformation is sufficiently fast for diffusive processes to limit the rate of interface advance. Assuming that the transformation kinetics is controlled by random nucleation, the explicit form of the growth-rate-limiting process determines the microstructure of the final product. Other quantities, such as transformation rate, mean grain size, and grain density, depend to some extent on the limiting mechanisms underlying the transformation.

The diffusion-controlled growth rate plays a fundamental role in a wide range of crystallization processes, especially in partitioning transformations. In a general sense, the kinetics of the grain growth is controlled by the diffusion of the slower species which must be included in, or expelled out of, the transformed phase. In most cases primary crystallization of amorphous materials falls into this picture and, therefore, an accurate knowledge of the diffusion process and the induced kinetics is essential in order to describe the properties of the microstructures developed in the crystallization process.

The diffusion process around an isolated growing grain is well established; see, for instance, Ref. 1. However, the diffusion around isolated grains cannot give a description of the interference between diffusion fields of neighbor grains at advanced stages of the phase transformation, which follows a nucleation and growth kinetics.

Nucleation and growth kinetics which results in the calculation of the volume fraction transformed at a given time is well established by the Kolmogorov-Johnson-Mehl-Avrami (KJMA) theory. ${ }^{2-6}$ KJMA's theory considers randomly distributed active nucleation sites which grow to form grains and during the growing process may collide with other grains of neighbor sites. To summarize, let us define a control volume $V_{0}$ where the growing phase occupies a volume $V(t)$; the transformed volume fraction is then $X(Y, t)=V(t) / V_{0}$. Here $Y$ is any of the macroscopic variables which may influence the kinetics. Avrami introduces the concept of extended volume $\widetilde{V}(t)$ as the volume that the growing grains would occupy neglecting impingement:

$$
\frac{d V}{d \widetilde{V}}=\frac{V_{0}-V}{V_{0}}
$$

This relationship means that the probability of each grain finding an untransformed volume to continue growing is randomly distributed. The KJMA theory gives an adequate description of the kinetics of any nucleation and growth transformation because it is based only on statistical considerations, being independent of the specific dynamics of the process. ${ }^{7}$ 
Equation (1.1) can be integrated under realistic conditions, provided that the nucleation rate $I(Y, t)$ and the growth rate $G(Y, t)$ are known. Several authors have performed these kind of calculations. ${ }^{8-11}$

Recently, Crespo and Pradell ${ }^{12}$ have proposed an extension of the Avrami's theory in order to calculate not only the transformed volume fraction but also the grain size distribution developed. This model has been tested against Monte Carlo simulations, showing excellent agreement.

In this paper, the above model is used and conveniently modified in order to describe diffusion-controlled grain growth in primary crystallization processes. In Sec. II A we summarize the features of the previous model. ${ }^{12}$ In Sec. II B radius-dependent grain growth is studied, and results obtained are applied to diffusion-controlled grain growth in Sec. II C. Overlapping of diffusion fields is considered in Sec. II D. Comparison with experimental data is also presented. Sec. III is devoted to the analysis of the grain size distributions obtained in each case and their properties. Concluding remarks are presented in Section IV.

\section{MODELING OF DIFFUSION-CONTROLLED GRAIN GROWTH}

\section{A. Mean field integration of Avrami's model}

As mentioned, Crespo and Pradell ${ }^{12}$ have proposed a mean-field model in order to calculate the grain size distribution obtained after nucleation and growth. The essential feature of this model is that it assumes that Eq. (1.1) can be applied in differential form to any grain population with a well-defined average radius. The main features of this model are now summarized.

The model is written in terms of reduced variables, which are presented as lowercase letters while dimensional variables are presented in as uppercase letters. A unit length $\eta$ is defined to make calculations dimensionless, i.e., the resolution length of a suitable observation method, and space dimensions are reduced by $\eta$. Time is also scaled by imposing the condition that the growing speed in the reduced system ( $g$ ) be unity. Then the time unit $\tau$ is defined by

$$
\eta=\int_{t}^{t+\tau(t)} G\left(Y, t^{\prime}\right) d t^{\prime}
$$

and, consequently, the reduced nucleation rate is

$$
i(t)=\eta^{3} \int_{t}^{t+\tau(t)} I\left(Y, t^{\prime}\right) d t^{\prime} .
$$

Let us assume that new nuclei have an average initial radius $R_{\epsilon}$; in the reduced system it becomes $r_{\epsilon}$, given by

$$
r_{\epsilon}=\frac{R_{\epsilon}}{\eta} .
$$

Extended and real populations by unit volume are defined, and their evolution is followed at unit time steps. Extended populations $\widetilde{n_{j, k}}$ are calculated assuming that grains grow in isolation. Due to the particular coordinate system chosen, its evolution is

$$
\begin{gathered}
\tilde{n}_{\epsilon, k+1}=i_{k}, \\
\tilde{n}_{j+1, k+1}=\tilde{n}_{j, k}, \quad j>\epsilon,
\end{gathered}
$$

where $\widetilde{n}_{j, k}$ is the extended population of radius $r_{j}$ per unit volume at time $t_{k}$, and $i_{k}=i\left(t_{k}\right)$. Note that all the grains nucleated at time $t_{k}$ are included in the population $\widetilde{n}_{\epsilon, k+1}$, thus having a finite number of populations which grow at unit speed. The extended transformed volume, coming from a unit volume, is easily written as

$$
\widetilde{v_{k}}=\sum_{j} \widetilde{n_{j, k}} \frac{4}{3} \pi r_{j}^{3} .
$$

Actual grain populations $n_{j, k}$ do grow at a slower speed than extended populations due to grain collisions. Our purpose is to calculate those populations explicitly. Therefore, an extension of the KJMA model is needed. The model is built in the following structure. Impingement between grains implies that only a fraction of the grains belonging to each population of radius $r_{j}$ at time $t_{k}$ is able to grow enough to have radius $r_{j+1}$ at time $t_{k+1}$. We define this fraction as $\alpha_{k}$, and postulate that this fraction is independent of the grain radius $r_{j}$ because it is only related to the probability of finding an untransformed volume near to a grain. This allows us to write equations equivalent to Eqs. (2.4) for the actual populations $n_{j, k}$ :

$$
\begin{gathered}
n_{\epsilon, k+1}=\left(1-\alpha_{k}\right) n_{\epsilon, k}+i_{k}\left(1-v_{k}\right), \\
n_{j+1, k+1}=\left(1-\alpha_{k}\right) n_{j+1, k}+\alpha_{k} n_{j, k}, \quad j>\epsilon,
\end{gathered}
$$

where $v_{k}$ is the transformed volume by unit volume, defined as

$$
v_{k}=\sum_{j} n_{j, k} \frac{4}{3} \pi r_{j}^{3} .
$$

The value of $\alpha_{k}$ is determined by imposing the condition that the volume differentials (extended and actual) satisfy Avrami's equation (1.1), which in this formulation is written as

$$
\frac{v_{k+1}-v_{k-1}}{\widetilde{v_{k+1}}-\widetilde{v}_{k-1}}=1-v_{k}
$$

Equations (2.4), (2.6), and (2.8) give an iterative method to obtain the grain size distributions present in the materials. The initial conditions for the integration are

$$
\begin{aligned}
& n_{j, 0}=0, \quad j=1, M, \\
& \tilde{n}_{j, 0}=0, \quad j=1, M,
\end{aligned}
$$

where $M$ is the arbitrarily large number of populations simulated. At each integration step Eqs. (2.4) are used to calculate the new values of the extended populations. Then the value of $\alpha_{k}$ is determined from Eqs. (2.6) and (2.8) by using a Newton-Rapson method. The model has been tested against Monte Carlo simulations, giving excellent results; see Ref. 12. We refer to this model as the mean-field constant-scale (MFC) model because the grain radius scale is homogeneous. 


\section{B. Modeling of radius-dependent grain growth}

Most of the nucleation and growth transformations are driven by a growth speed dependent on the grain radius, i.e., $G=G(Y, t, r)$. The MFC model is not able to reproduce such processes because the radius dependence of the growth rate breaks the grain radius scale homogeneity which is underlying the MFC model. For that reason, in this section we present a modification of the MFC model which is able to deal with the particular case of time-independent radiusdependent growth rates, i.e., $G=G(r)$, which we will refer to as the mean-field variable-scale (MFV) model. In particular, this model will apply to diffusion-controlled grain growth.

In order to adapt the MFC model to radii-dependent growth, it is necessary to define a variable radius length scale, by imposing

$$
g\left(r_{k}\right)=1 \quad \forall k
$$

This is achieved by observing that the radius of an isolated grain, nucleated at $t=t_{0}$ with an initial radius $R_{\epsilon}$, is a continuous function of $\left(t-t_{0}\right)$ given by

$$
R(t)=R_{\epsilon}+\int_{t_{0}}^{t} G\left(t^{\prime}-t_{0}\right) d t^{\prime}
$$

This is a universal function, in the sense that the radius of all grains has the same time dependence after nucleation. Then, for a given value of $\eta$ we have

$$
r\left(t-t_{0}\right)=r_{\epsilon}+\frac{1}{\eta} \int_{0}^{t-t_{0}} G\left(t^{\prime}\right) d t^{\prime}
$$

which establishes that there is a univocal relationship between the grain radius and the time elapsed since nucleation. Therefore, the value of $\tau$ is defined by

$$
\eta=\int_{0}^{\tau} G\left(t^{\prime}\right) d t^{\prime}=r(\tau)-r_{\epsilon} .
$$

Once defined $\tau$, the values of $r_{k}$ are simply

$$
r_{\epsilon+k}=r(k \tau),
$$

which automatically satisfy Eq. (2.10).

Expression (2.14) ensures that extended populations $\tilde{n}_{j}^{k}$ will always transform into $\tilde{n}_{j+1}^{k+1}$ for any values of $j$ and $k$. This property also implies the need of a time independence of $G$, because changing values of $G$ would imply the redefinition of the radius scale, thus invalidating the definition of populations.

Under the above condition, Eqs. (2.4), (2.6), and (2.8) apply without any correction, conserving their meaning. Calculation of the actual grain populations according to this definition is achieved straightforwardly by using the same computation strategy as in the MFC model.

This MFV model allows us to study the crystallization behavior of a primary transformation.

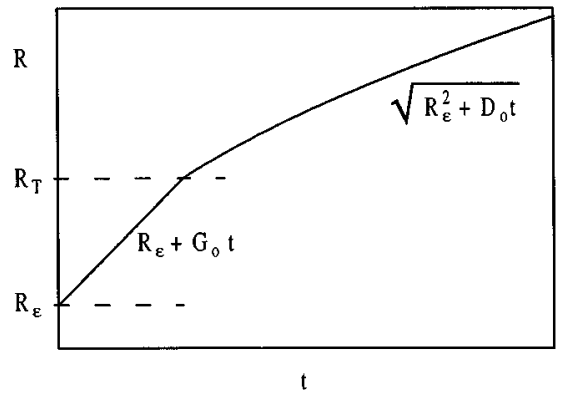

FIG. 1. Plot of $R(t)$ in a interface-plus-diffusion-controlled grain growth process.

\section{Application: Interface plus diffusion controlled grain growth}

We will consider a particular case of a transformation driven by homogeneous nucleation of grains (of spherical shape) of critical radius $R_{\epsilon}$ whose initial growth occurs at a constant rate up to a threshold radius $R_{T}$, further growth being diffusion controlled. ${ }^{13}$ Under steady-state conditions the growth velocity will become

$$
\frac{d R}{d t}=D \frac{C^{*}-C_{0}}{C^{*}-C_{x t}} \frac{1}{R},
$$

where $D$ is the diffusion coefficient for the slowest solute that piles up ahead of the interface, and $C_{x t}, C^{*}$, and $C_{0}$ stand, respectively, for the concentration of that solute inside the grain, at the grain interface, and at very large distances compared to the radius of the grain.

In this section we ignore the interference between different growing grains that arises from the competition for the available excess solute, since it becomes important only when the diffusion fields of two grains begin to overlap to an appreciable extent. The more general problem of interference is treated in the next section. Thus, as a first approximation, isolated grains grow in an infinite matrix and $C$ has the initial value $C_{0}$ of the initial phase. We will define an effective diffusion coefficient $D_{0}$ as

$$
D_{0}=D \frac{C^{*}-C_{0}}{C^{*}-C_{x t}},
$$

and then

$$
\frac{d R}{d t}=\frac{D_{0}}{R}
$$

In order to select between the two growth mechanisms, the continuity in radius size is imposed, resulting in

$$
R(t)= \begin{cases}R_{\epsilon}+G_{0} t, & R(t) \leqslant R_{T}, \\ \sqrt{R_{\epsilon}^{2}+D_{0} t} & \text { otherwise, }\end{cases}
$$

where $G_{0}$ is the interface controlled growth rate and the threshold radius $R_{T}$ is

$$
R_{T}=\frac{D_{0}}{G_{0}}-R_{\epsilon} .
$$

Figure 1 shows the behavior of $R(t)$. 


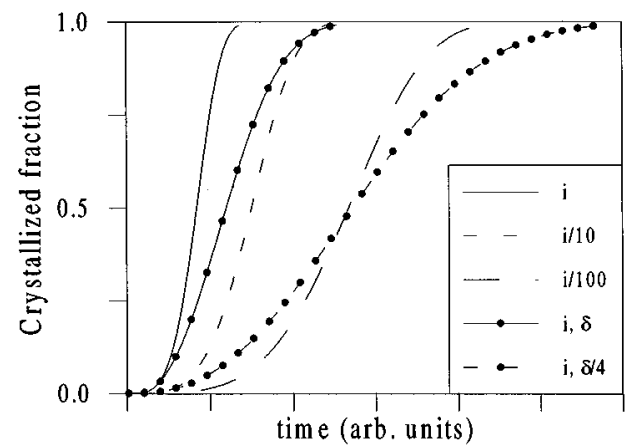

FIG. 2. Plot of the crystalline fraction vs time for a given growth rate $g$ and reduced nucleation rate $i=4 \times 10^{-8}, i / 10$, and $i / 100$ without diffusion, and for the same values of $i$ and $g$ with diffusion obtained when $\delta=1.2$ and $\delta / 4$.

The length scale of the MFV model applied to this case is written in reduced units as

$$
r_{j}=\left\{\begin{array}{l}
j, \quad j \leqslant R_{T} / \eta, \\
\sqrt{r_{\epsilon}^{2}+(j-\epsilon) \delta} \quad \text { otherwise },
\end{array}\right.
$$

where $\delta$ is the reduced diffusion coefficient defined by

$$
\delta=\frac{D_{0}}{G_{0} \eta}
$$

and $R_{T} / \eta=\delta-r_{\epsilon}$ in reduced units.

We now illustrate the effect of the introduction of the diffusion in the grain growth. Figure 2 plots the evolution of the crystalline fraction versus time for a given growth rate $g$ and three different values of the reduced nucleation rate, namely, $i=4 \times 10^{-8}$, and $i / 10$, and $i / 100$, without diffusion. Moreover, two diffusion-controlled processes obtained for the same values of $g$ and $i$, and reduced diffusion coefficients $\delta=1.2$ and $\delta / 4$, are presented. As a general trend, both diffusion and reduction of the nucleation rate retard the crystallization, but in a different way. Therefore, the introduction of the diffusion mechanism provides more flexibility for modeling grain size populations in transformations for which the KJMA theory is applicable.

We can also visualize the relative importance of the two mechanisms, nucleation rate and diffusion, by plotting in Fig. 3 the number of grains versus time for a given value of $g$ and different values of $i$ and $\delta$. Although reducing the nucleation rate and introducing diffusion both delay the transition, the effect on the grain density is the opposite: Reducing the nucleation rate drastically reduces the total number of grains, while the introduction of diffusion increases it.

The model of grain growth used assumes that diffusion takes place only after the grain reaches a threshold radius $R_{T}$. Therefore, diffusion-controlled growth takes place above the time needed for the first nuclei to reach $R_{T}$. This is also shown in Fig. 3, where the relative fractions of grains growing either by interface or by diffusion are also plotted. At increasing time, diffusion becomes the dominant mechanism of grain growth. The average grain radius also shows these differences, as shown in Fig. 4. Without diffusion, the average grain size increases as the nucleation rate $i$ de-

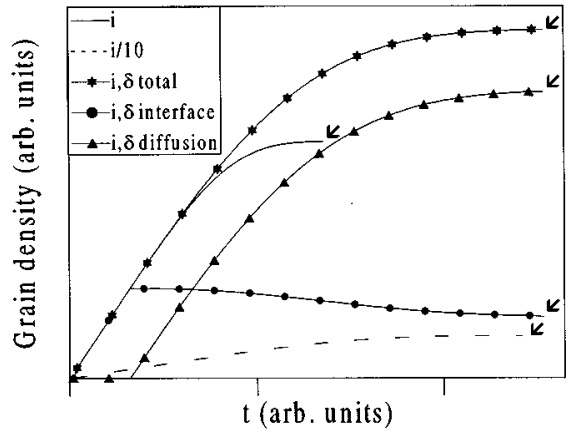

FIG. 3. Plot of the grain density vs time obtained for a given value of $g$ and $i=4 \times 10^{-8}$ (solid line) and $i / 10$ (dashed line) without diffusion. Plot of the total (stars line), interface-controlled (circles line), and diffusion-controlled (triangles line) grain density in an interface-plus-diffusion-controlled process with the same values of $i$ and $g$ and a reduced diffusion coefficient $\delta=1.2$.

creases, but it is reduced as soon as diffusion plays a role. The effect on the grain size distribution shape will be discussed in Sec. III.

We will apply this model to the experimental data obtained for the primary crystallization of $\alpha-\mathrm{Fe}(\mathrm{Si}) \mathrm{DO}_{3}$ structure in a $\mathrm{Fe}_{73.5} \mathrm{CuNb}_{3} \mathrm{Si}_{17.5} \mathrm{~B}_{5}$ amorphous alloy. As is well known, ${ }^{14-18}$ nanocrystallization is promoted by the presence of $\mathrm{Nb}$ atoms which limit the grain size. $\mathrm{Nb}$ is expelled from the crystalline structures, generating a diffusion layer in the surrounding matrix. ${ }^{19}$ The homogeneous nucleation frequency and interface-controlled growth dependence on temperature are evaluated by the classical theory, ${ }^{20}$ and the diffusion coefficient $D_{0}$, corresponding to $\mathrm{Nb}$ atoms, is assumed to follow an Arrhenius temperature dependence. The values of the several relevant quantities are shown in Table I for specific temperatures. Experimental data on $X(t)$ were obtained under isothermal transformation of the sample heat treated in a differential scanning calorimetry (DSC) cell. ${ }^{21,22}$

The isothermal evolution of the transformed fraction for three different temperatures $\left(490,500\right.$, and $\left.510{ }^{\circ} \mathrm{C}\right)$ is obtained by the integration of the MFC model for interfacecontrolled growth and the MFV model for interface-plusdiffusion-controlled growth. Figure 5 shows the comparison

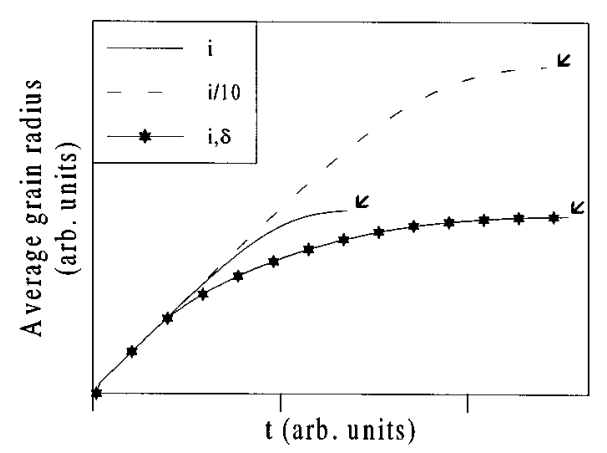

FIG. 4. Plot of the average grain radius vs time obtained for a given value of $g$ and $i=4 \times 10^{-8}$ (solid line) and $i / 10$ (dashed line) without diffusion, and with the same values of $i$ and $g$ and a reduced diffusion coefficient $\delta=1.2$ (stars line). In all cases the arrows indicate the end of the primary crystallization. 
TABLE I. Parameters used in the calculation of the nucleation rate $I$, the growth rate $G$, and the diffusion coefficient $D_{0}$ for the $\mathrm{Fe}_{73.5} \mathrm{CuNb}_{3} \mathrm{Si}_{17.5} \mathrm{~B}_{5}$ amorphous alloy.

\begin{tabular}{ccccc}
\hline \hline $\begin{array}{c}T \\
\left({ }^{\circ} \mathrm{C}\right)\end{array}$ & $\begin{array}{c}R_{\epsilon} \\
(\mathrm{nm})\end{array}$ & $\begin{array}{c}G_{0} \\
\left(\mathrm{~cm} \mathrm{~s}^{-1}\right)\end{array}$ & $\begin{array}{c}I \\
\left(\mathrm{~cm}^{-3} \mathrm{~s}^{-1}\right)\end{array}$ & $\begin{array}{c}D_{0} \\
\left(\mathrm{~cm}^{2} \mathrm{~s}^{-1}\right)\end{array}$ \\
\hline 490 & 1.106 & $3.2417 \times 10^{-09}$ & $1.4112 \times 10^{+15}$ & $1.6826 \times 10^{-15}$ \\
500 & 1.106 & $6.0970 \times 10^{-09}$ & $3.5303 \times 10^{+15}$ & $2.6611 \times 10^{-15}$ \\
510 & 1.106 & $1.0993 \times 10^{-08}$ & $8.3671 \times 10^{+15}$ & $4.1597 \times 10^{-15}$ \\
\hline \hline
\end{tabular}

between experimental data ${ }^{21,22}$ and the values calculated with the two models above mentioned. By convention, the transformed volume fraction is taken to be $X=1$ at the end of the primary crystallization, while a volume fraction $1-\gamma$ of the specimen remains untransformed.

Close observation of Fig. 5 indicates that the computed volume fraction for both models fits the experimental data at the onset of the transformation. However, systematic overestimation of the transformed fraction occurs when considering only interface-controlled growth. Overestimation, compared to experimental data, likewise appears in the computed values when the interface-plus-diffusion-controlled growth is used, but it occurs mainly at the late stages of the transformation. These results suggest that, in the crystallization process considered, the onset is driven by homogeneous nucleation- and interface-controlled growth, while the diffusion-controlled growth dominates further crystallization stages. However, in this particular alloy some further mechanism is retarding the crystallization.

\section{Model of overlapping diffusion fields}

The interference between nearby growing grains is part of the diffusion problem and results in a growth rate that gradually becomes zero. ${ }^{23}$ This is due to the fact that the concentration profile in the matrix is altered by the presence of nearby grains, becoming time dependent, as sketched in Fig. 6. In a general sense, this corresponds to the situation where the growth of the grains modifies their surroundings, i.e., in

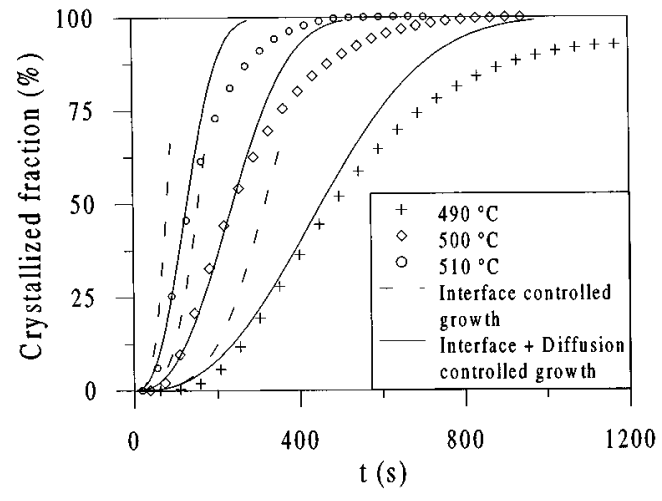

FIG. 5. Experimentally measured transformed volume fraction vs time for the isothermal crystallization of $\mathrm{Fe}_{73.5} \mathrm{CuNb}_{3} \mathrm{Si}_{17.5} \mathrm{~B}_{5}$ metallic alloy at several temperatures. Calculated crystalline fractions for interface-controlled growth (dotted line) and with the introduction of the diffusion-controlled process (solid line) are also shown for the three temperatures.

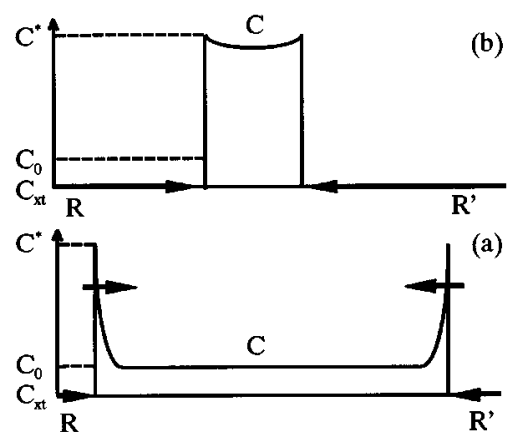

FIG. 6. Scheme of the concentration profile between grains at the beginning (a) and near the end (b) of the crystallization process, showing the effect of the overlapping diffusion fields.

partitioning transformations. The concentration gradient at the grain interface is fortunately quite insensitive to the precise manner in which we specify the boundary conditions far away from one grain. ${ }^{24}$ At the final stages of the transformation, one expects $\left|C^{*}-C\right| \ll\left|C^{*}-C_{x t}\right|$. In this case, the interface will move sufficiently slowly for the steady-state equation (2.15) to be appropriate. However, the presence of neighbor grains growing simultaneously will increase $C$, which instead of remaining constant (and equal to $C_{0}$ ), as assumed in the first approximation, will vary with time and depending on the microstructure developed.

The main problem in order to take this effect into account is that spherical symmetry is lost due to the random distribution of grains around a given grain of radius $R$. Moreover, Eq. (2.15) is obtained under the assumption that far away from the growing grain the concentration of solute is known. In the present case, we consider that the grains are still growing in isolation by substituting the effect of the rest of the growing grains for an increase in the average concentration $C(t)$ of solute in the matrix far away from the grain at any given time. That is, we will substitute the concentration inhomogeneity, due to individual grains, for the mean concentration field $C(t)$. Therefore, Eq. (2.15) will still be applicable, replacing $C_{0}$ by $C(t)$. In order to evaluate this timedependent concentration of solute, a mass balance at time $t$ has to be written, yielding

$$
\gamma X(t) C_{x t}+[1-\gamma X(t)] C(t)=C_{0} .
$$

The value of $C(t)$ is then

$$
C(t)=\frac{C_{0}-\gamma X(t) C_{x t}}{1-\gamma X(t)} .
$$

Introducing this value in Eq. (2.15) in place of $C_{0}$, we obtain after some transformation

$$
\frac{d R}{d t}=\left(1-\frac{1-f_{x t}}{1-f_{0}} \gamma X(t)\right) \frac{1}{1-\gamma X(t)} \frac{D_{0}}{R} .
$$

Here $f_{0}=C_{0} / C^{*}, f_{x t}=C_{x t} / C^{*}$, and $\gamma$ are assumed constants which might be determined experimentally. It is easy to see that at the beginning of the transformation, that is, at the limit $X(t) \rightarrow 0$, the growth speed is the same as defined by Eq. (2.17). 
In order to simplify the notation, we will define the coefficient

$$
\sigma(X(t))=\left(1-\frac{1-f_{x t}}{1-f_{0}} \gamma X(t)\right) \frac{1}{1-\gamma X(t)},
$$

so that

$$
\frac{d R}{d t}=\sigma(X(t)) \frac{D_{0}}{R} .
$$

Therefore, the radius evolution of a grain is still given by Eqs. (2.18) and (2.19), substituting $D_{0}$ by $\sigma(X(t)) D_{0}$. This produces a double effect on the growth protocol; on the one hand there is an additional reduction of the diffusioncontrolled growth rate as the transformed volume fraction increases, while on the other hand there is a reduction of the threshold radius at which diffusion begins to control the growth rate.

The introduction of this variable diffusion rate in the MFV model is performed by defining the same variable radius scale as in Eq. (2.20), and modifying Eqs. (2.4) and (2.6) as follows:

$$
\begin{gathered}
\tilde{n}_{\epsilon, k+1}=i_{k}, \\
\tilde{n}_{j+1, k+1}=\tilde{n}_{j, k}, \quad \sigma\left(X_{k}\right) \delta-r_{\epsilon}>j>\epsilon, \\
\tilde{n}_{j+1, k+1}=\left(1-\sigma\left(X_{k}\right) \frac{\delta}{r_{j+1}}\right) \tilde{n}_{j+1, k}+\sigma\left(X_{k}\right) \frac{\delta}{r_{j}} \tilde{n}_{j, k}, \\
\tilde{n}_{j+1, k+1}=\left[1-\sigma\left(X_{k}\right)\right] \tilde{n}_{j+1, k}+\sigma\left(X_{k}\right) \tilde{n}_{j, k}, \quad j>r_{T}, \\
n_{\epsilon, k+1}=\left(1-\alpha_{k}\right) n_{\epsilon, k}+i_{k}\left(1-V_{k}\right), \\
n_{j+1, k+1}=\left(1-\alpha_{k}\right) n_{j+1, k}+\alpha_{k} n_{j, k}, \quad \sigma\left(X_{k}\right) \delta-r_{\epsilon}>j>\epsilon, \\
n_{j+1, k+1}=\left(1-\alpha_{k} \sigma\left(X_{k}\right) \frac{\delta}{r_{j+1}}\right) n_{j+1, k}+\alpha_{k} \sigma\left(X_{k}\right) \frac{\delta}{r_{j}} n_{j, k}, \\
r_{T}>j>\sigma\left(X_{k}\right) \delta-r_{\epsilon}, \quad(2.28) \\
n_{j+1, k+1}=\left[1-\alpha_{k} \sigma\left(X_{k}\right)\right] n_{j+1, k}+\alpha_{k} \sigma\left(X_{k}\right) n_{j, k}, \quad j>r_{T} .
\end{gathered}
$$

Here, $X_{k}=X\left(t_{k}\right)$ and, consequently, $\sigma\left(X_{k}\right)$ are considered slowly varying variables; their variation during the integration step is neglected and they are recomputed at each iteration. It is worth noting that it is necessary to separate the grains of radius $\sigma(X(t)) \delta-r_{\epsilon}<r_{j}<r_{T}$ because they first grow at a constant rate, their radius scale being constant. Afterwards their growth is controlled by diffusion, and the growth rate becomes proportional to $1 / R$.

Let us evaluate the effect of the existence of overlapping diffusion fields in our previous example, of the primary rystallization of $\alpha-\mathrm{Fe}(\mathrm{Si}) \quad \mathrm{DO}_{3}$ structure in a $\mathrm{Fe}_{73.5} \mathrm{CuNb}_{3} \mathrm{Si}_{17.5} \mathrm{~B}_{5}$ amorphous alloy. In this particular case, it has been determined experimentally that $C_{x t}=0$. Mössbauer results indicate that primary crystallization ends approximately after annealing $4 \mathrm{~h}(1 \mathrm{~h})$, at $490{ }^{\circ} \mathrm{C}\left(510^{\circ} \mathrm{C}\right)$; so we accept that $X=1$ at this moment. Analysis of the

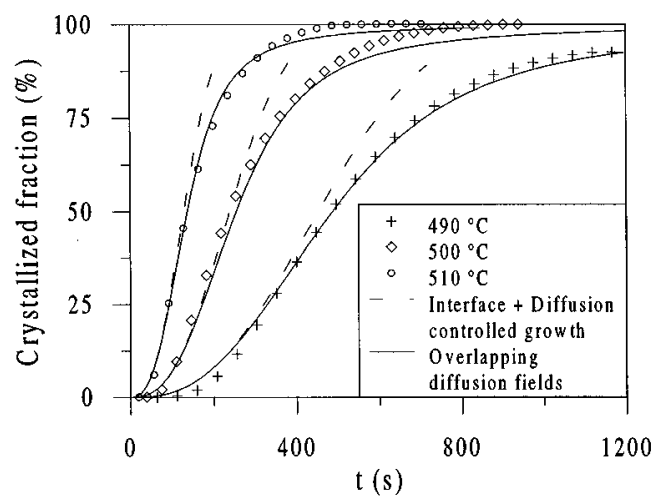

FIG. 7. Experimentally measured transformed volume fraction vs time for the isothermal crystallization of $\mathrm{Fe}_{73.5} \mathrm{CuNb}_{3} \mathrm{Si}_{17.5} \mathrm{~B}_{5}$ metallic alloy at several temperatures. The calculated crystalline fractions with the diffusion-controlled process (dotted line) and with the introduction of the overlapping diffusion model (solid line) are shown.

Mössbauer spectra also gives the value of $\gamma(T) X(t)$, obtaining $\gamma\left(490{ }^{\circ} \mathrm{C}\right)=0.60 \pm 0.05$ (Ref. 25) and $\gamma\left(510^{\circ} \mathrm{C}\right)$ $=0.65 \pm 0.05 .^{26}$ These parameters have not been determined at $500{ }^{\circ} \mathrm{C}$, but it seems plausible to accept that $\gamma\left(500{ }^{\circ} \mathrm{C}\right)$ $\simeq 0.62 \pm 0.05$ ). The only unknown parameter still undefined in Eq. (2.24) is $C^{*}$, which we will fix by imposing the condition that no diffusion gradients exist in the remaining matrix; that is, $C(t) \rightarrow C^{*}$ when $t \rightarrow \infty$. This results in

$$
\frac{C_{0}}{C^{*}}=f_{0}=1-\gamma
$$

With this assumption, no new adjustable parameters are introduced in the modeling of the growing speed.

The results of the integration of the MFV model using the growth rate defined by Eq. (2.24) and the above-mentioned assumptions are compared with experimental data in Fig. 7 for the annealing of the samples at the same temperatures $\left(490,500\right.$, and $\left.510^{\circ} \mathrm{C}\right)$. The agreement between computed and experimental data is excellent, always below the uncertainty of the experimental data $( \pm 10 \%)$.

\section{GRAIN SIZE DISTRIBUTIONS}

The final goal of the above formulations is to obtain a quantitative description of the resulting grain size distributions after controlled thermal treatments. ${ }^{12}$ The shape of the size distribution has substantial influence on most of the macroscopic physical properties of interest, i.e., magnetic coercitivity and susceptibility, electric resistance, etc. However, the parameters usually given in the description of a microstructure, namely, the grain density and the average grain radius, are quite insensitive to the real grain size distribution. Thus, the ability of the above models to obtain those distributions greatly increases the knowledge of the microstructure, which is the essential feature controlling the macroscopic properties. $^{27-30}$

Figures 8 and 9 show the computed grain size distributions after isothermal treatments of $\mathrm{Fe}_{73.5} \mathrm{Cu} \mathrm{Nb} \mathrm{Nb}_{3} \mathrm{Si}_{17.5} \mathrm{~B}_{5}$ at 490 and $510{ }^{\circ} \mathrm{C}$ for several crystallized fractions. Interfacecontrolled growth produces a broad grain size distribution, 


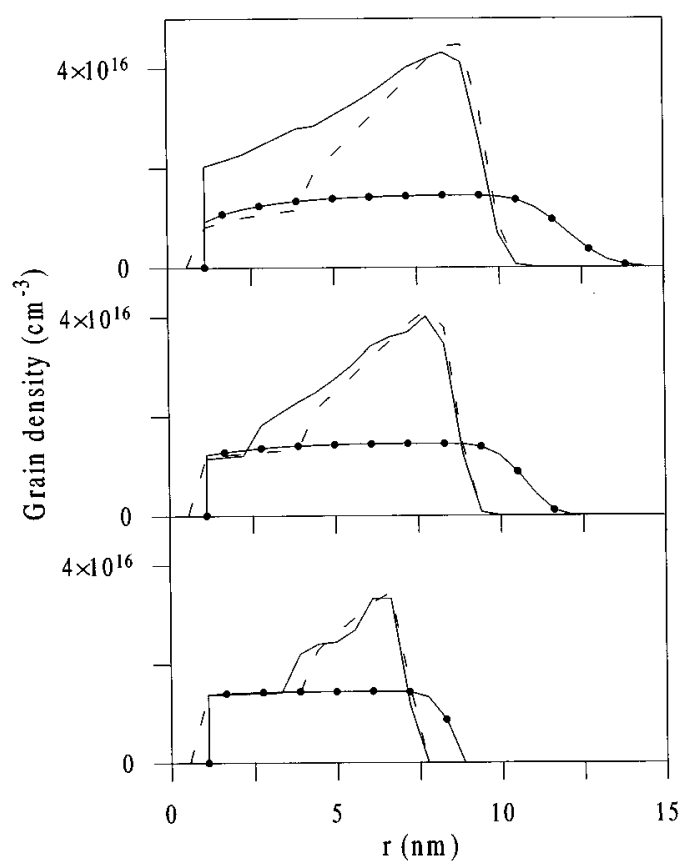

FIG. 8. Plot of the grain size distributions for isothermal annealing of $\mathrm{Fe}_{73.5} \mathrm{CuNb}_{3} \mathrm{Si}_{17.5} \mathrm{~B}_{5}$ at $490{ }^{\circ} \mathrm{C}$ at three different crystallized fractions, namely, $X=20 \%, 60 \%$, and $99 \%$. The dotted line shows the resulting distribution for the interface-controlled growth mechanism. The dashed line includes interface-plus-diffusion-controlled growth. The solid line shows the result of interface plus overlapping diffusion fields growth.

showing a flat profile with a similar number of grains for all radii below the maximum. The introduction of a diffusion mechanism dramatically changes the shape of the distribution, giving a main asymmetric peak. The discontinuity observed on the left side of the peak appears at the threshold radius, where diffusion becomes the controlling mechanism of grain growth. The shape of the discontinuity is due to the discontinuous model of growing mechanism used, which assumes continuity in the radius growth but not in the growing speed, being only a first approach of the true growing mechanism. In fact, a smooth transition between interface- and diffusion-controlled growth is expected, and more sophisticated models may offer a more realistic description. However, the effect of this improvement of the growing description should not affect the main trends of the shape of the distribution.

The most interesting features of the introduction of a diffusion-controlled growing mechanism are the refinement of the microstructure and the reduction of the maximum grain size, closely related to the asymmetry observed in the shape of the distribution.

The delay of the crystallization given by the introduction of the presence of overlapping diffusion fields gives a further reduction of the maximum grain radius and results in an accumulation of grains at small radiis, giving a more asymmetric grain size distribution.

The main features of the grain size distribution are the same at higher annealing temperatures, but an extra reduction of the average grain size and maximum grain size and an increase of the total number of grains and peak maximum are also observed. Therefore, a sharper distribution with a

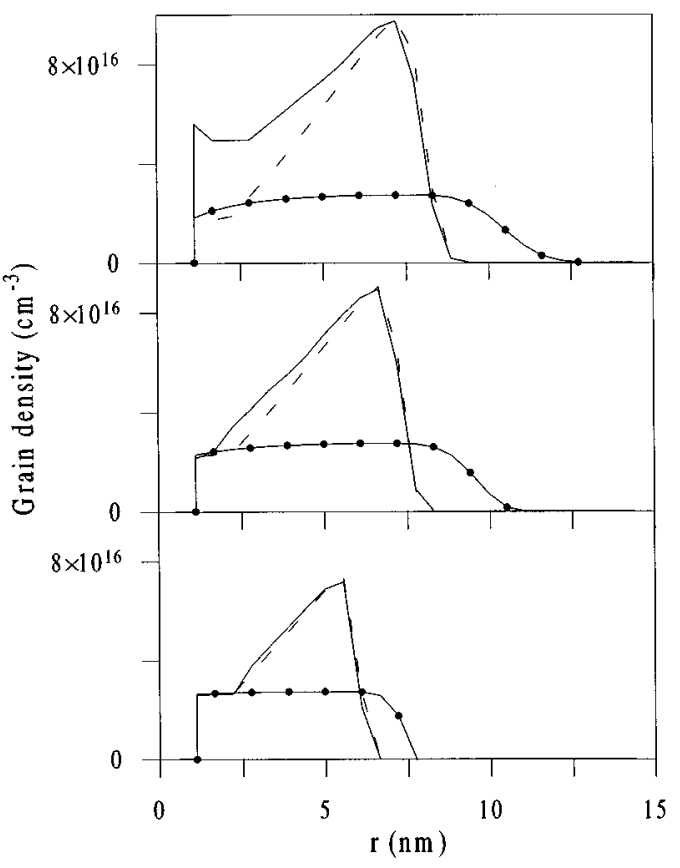

FIG. 9. Plot of the grain size distributions for isothermal annealing of $\mathrm{Fe}_{73.5} \mathrm{CuNb}_{3} \mathrm{Si}_{17.5} \mathrm{~B}_{5}$ at $510{ }^{\circ} \mathrm{C}$ at three different crystallized fractions, namely, $X=20 \%, 60 \%$, and $99 \%$. The dotted line shows the resulting distribution for the interface-controlled growth mechanism. The dashed line includes interface-plus-diffusion-controlled growth. The solid line shows the result of interface plus overlapping diffusion fields growth.

smaller average radius will be obtained with a higher number of grains while increasing temperature.

The refinement of the distribution produced by the diffusion process may also be observed in Fig. 10, where the average grain size and maximum grain size of the distribution are plotted as a function of the annealing temperature for the overlapping diffusion field model, and are compared with the interface-controlled growth results. Although the average grain size is only slightly reduced, it becomes evident that the maximum grain size is strongly cut down because of the

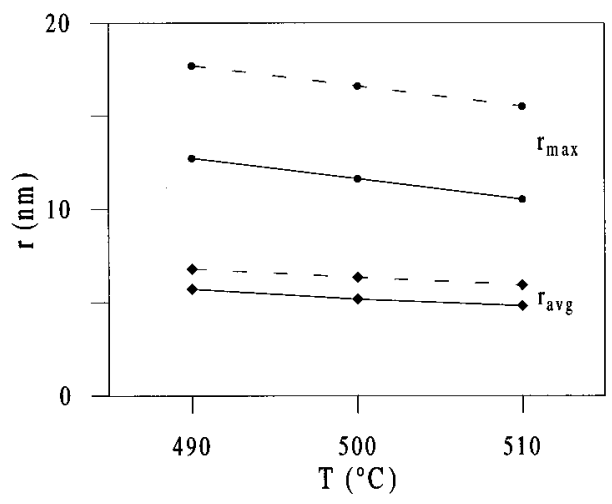

FIG. 10. Dependence of the average (diamonds) and maximum (circles) grain size with annealing temperature of $\mathrm{Fe}_{73.5} \mathrm{CuNb}_{3} \mathrm{Si}_{17.5} \mathrm{~B}_{5}$ for the overlapping diffusion field model (solid line) and the interface-controlled growth (dashed line). Results for the diffusion model are indistinguishable from the average diffusion field model. 


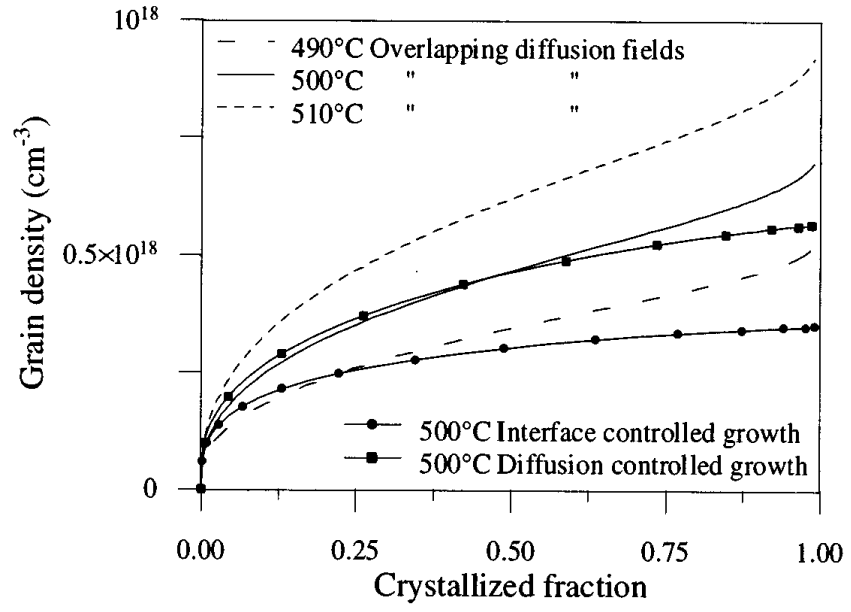

FIG. 11. Plot of the grain density vs crystallized fraction in $\mathrm{Fe}_{73.5} \mathrm{CuNb}_{3} \mathrm{Si}_{17.5} \mathrm{~B}_{5}$.

diffusion, and it is additionally reduced by an increase in the annealing temperature. Results for the diffusion model are indistinguishable from the overlapping diffusion field model and so are not plotted.

The above statements greatly coincide with the empirical knowledge about the suitable annealing procedures to obtain small grain sizes. Effectively, it is well known that better macroscopical properties are obtained by annealing at higher temperatures, provided that no secondary crystallization begins.

Another critical parameter of the obtained grain size distribution is the grain density. It is shown in Fig. 11 for the three models studied above and the three temperatures, as a function of the crystallized fraction. Comparing the interface-controlled growth and the diffusion-controlled growth for a given temperature, the effect of the diffusion mechanism is to increase the grain density by a factor of about 3/2. Furthermore, the overlapping diffusion field model still gives a further increase in the grain density, due essentially to the additional reduction of the growing speed for larger grains.

In the final stages of the crystallization process, for crystallized fractions over 0.9 , the overlapping diffusion field model gives rise to an additional increase in the total grain number. This increase is due to the fact that the nucleation rate is considered constant in the whole process. However, the change in composition of the remaining amorphous phase should result in a reduction of the nucleation rate at the final stages of the crystallization. ${ }^{31}$ This has not been considered in the present model, and will result in a smoother increase of the total number of grains.

The relatively important number of grains of small sizes responsible for the marked asymmetry of the grain size distributions will, in fact, have a low effect on any extensive macroscopical property. In fact, considering the fraction of volume occupied by each population of a given radius, the populations with small radii will also make a small contribution to the total crystallized volume. The volume fraction distribution, obtained by plotting the volume occupied by

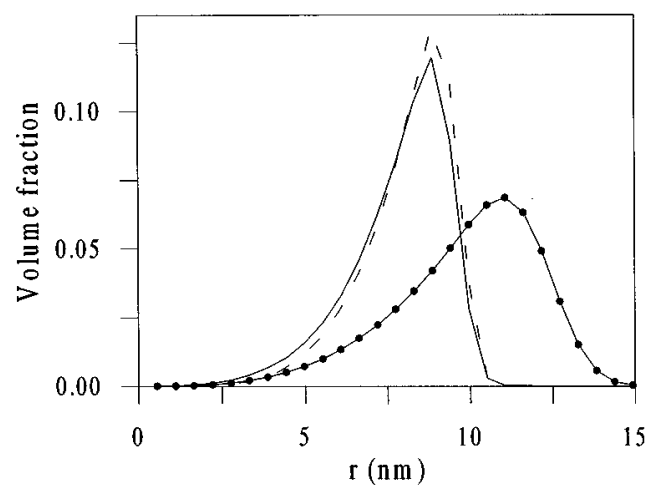

FIG. 12. Volume fraction occupied by each population after completion of primary crystallization $(X=1)$ for a sample of $\mathrm{Fe}_{73.5} \mathrm{CuNb}_{3} \mathrm{Si}_{17.5} \mathrm{~B}_{5}$ annealed at $490{ }^{\circ} \mathrm{C}$ at the end of the primary crystallization. The dotted line shows the resulting distribution for the interface-controlled growth mechanism. The dashed line includes interface-plus-diffusion-controlled growth. The solid line shows the result of interface plus overlapping diffusion fields growth.

each population, is presented in Fig. 12, and shows that there is only a narrow range of grain sizes having appreciable contributions to any extensive macroscopical property. What is also important is the main change induced by the diffusion mechanism controlling the growth rate, namely, a clear decrease in the size $(\sim 30 \%)$ of the relevant populations. This distribution is directly comparable with the experimental data obtained by indirect Fourier transform of small angle neutron scattering (SANS) and small angle x-ray scattering (SAXS) spectra. These methods give volume fraction distributions, but not absolute measurements of the number of grains. Measurements of a crystallized material of a similar composition $\left(\mathrm{Fe}_{73.5} \mathrm{CuNb}_{3} \mathrm{Si}_{15.5} \mathrm{~B}_{7}\right),{ }^{32}$ annealed for $1 \mathrm{~h}$ at $550{ }^{\circ} \mathrm{C}$, give an average grain size of about $5 \mathrm{~nm}$ and a maximum grain size of about $8 \mathrm{~nm}$. The shape of the obtained distribution is very close to those computed with the interface-plus-diffusion-controlled growth model.

The developed microstructure may also be evaluated by transmission electron microscopy (TEM) analysis. However, it has some limitations both in resolution and statistics. On the one hand, the resolution of the TEM spot limits the minimum size of a detectable grain, thus avoiding the detection of smaller grains, as well as in the separation between contiguous grains. On the other hand, the finite thickness of the sample is responsible for grain overlapping. Both facts can lead to an underestimation of the total number of grains. Moreover, as shown by several authors, ${ }^{33}$ the shape of the TEM grain size distribution also depends on the tracks of the cut grains on the two surfaces of the sample, which may also be evaluated as in Ref. 12. The observed grain size distribution becomes a mixture between the grain size distribution and the surface grain size distribution. As an example, the final surface distribution for the sample annealed at $490{ }^{\circ} \mathrm{C}$ and $510{ }^{\circ} \mathrm{C}$, computed with the overlapping diffusion fields model following Ref. 12, is shown in Figs. 13 and 14. The shape of this distribution shows a more rounded main peak than the grain size distribution. 


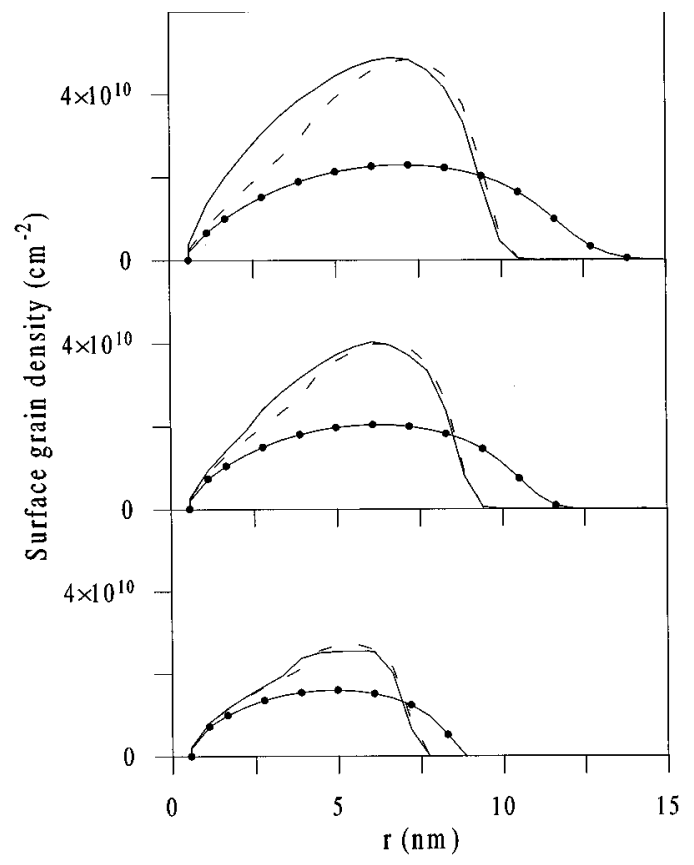

FIG. 13. Final surface grain size distribution of $\mathrm{Fe}_{73.5} \mathrm{CuNb}_{3} \mathrm{Si}_{17.5} \mathrm{~B}_{5}$, isothermally annealed at $490{ }^{\circ} \mathrm{C}$, computed by using the overlapping diffusion field model at three different crystallized fractions, namely, $x=20 \%, 60 \%$, and $99 \%$. The dotted line shows the resulting distribution for the interface-controlled growth mechanism. The dashed line includes interface-plusdiffusion-controlled growth. The solid line shows the result of interface plus overlapping diffusion fields growth.

TEM analysis of the samples after $2 \mathrm{~h}$ of annealing at $490{ }^{\circ} \mathrm{C}$ (Refs. 22 and 25) gave an average grain radius of about $5 \mathrm{~nm}$ and a maximum grain radius of about $15 \mathrm{~nm}$, in good agreement with the calculated results for the overlapping diffusion fields model, namely, 5.7 and $12.7 \mathrm{~nm}$. Experimental estimation of the final number of grains by unit surface and volume gives values of about $4 \times 10^{11}$ and $2 \times 10^{17} \mathrm{~cm}^{-3}$, respectively, which are in excellent agreement with the computed values of $5.9 \times 10^{11}$ and $5.2 \times 10^{17}$ $\mathrm{cm}^{-3}$.

\section{CONCLUSIONS}

A mean-field model for nucleation and diffusioncontrolled growth processes in primary crystallization has been developed which allows one to evaluate the microstructure development by computing the grain size-distribution. In a general sense, this corresponds to the situation where the growth of the grains modifies their surroundings, which is the case in partitioning transformations. The body of the model is a generalization of the already developed ${ }^{12}$ nucleation and growth kinetics model for evaluating grain size distributions, conveniently modified to include sizedependent crystal growth.

Comparison between the calculations performed in the case of interface-controlled growth and diffusion-controlled growth (without overlapping diffusion fields) is given, and the relative importance of the nucleation and growth mecha-

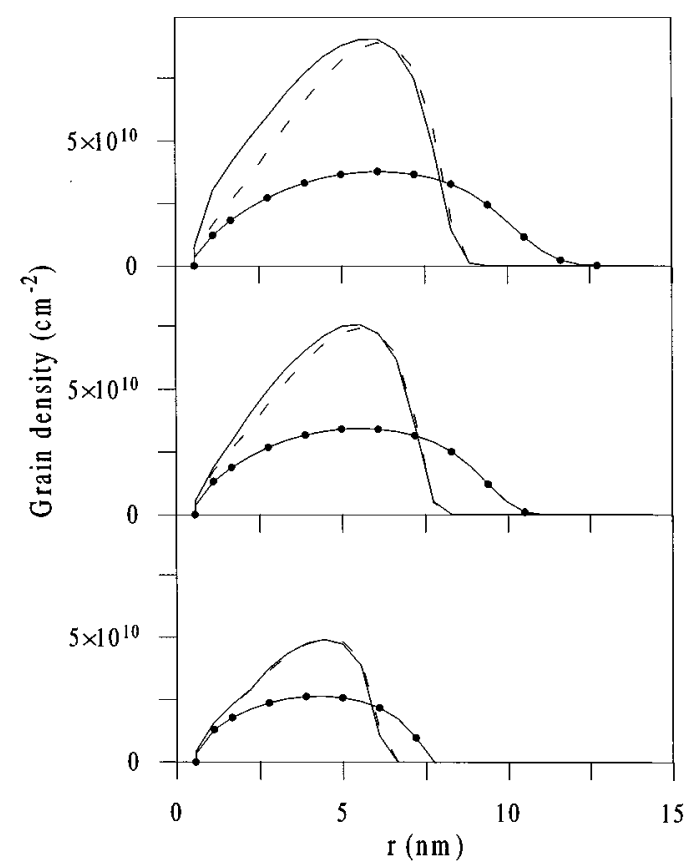

FIG. 14. Final surface grain size distribution of $\mathrm{Fe}_{73.5} \mathrm{CuNb}_{3} \mathrm{Si}_{17.5} \mathrm{~B}_{5}$, isothermally annealed at $510{ }^{\circ} \mathrm{C}$, computed by using the overlapping diffusion field model at three different crystallized fractions, namely, $x=20 \%, 60 \%$, and $99 \%$. The dotted line shows the resulting distribution for the interface-controlled growth mechanism. The dashed line includes interface-plusdiffusion-controlled growth. The solid line shows the result of interface plus overlapping diffusion fields growth. Fig. 8 .

nisms in both cases is analyzed. Extensive analysis of thedata is presented with respect to the macroscopical and microscopical property evolution.

As a main result it has been demonstrated that the introduction of diffusion mechanisms is crucial for understanding the microstructure development. Consideration of the overlapping diffusion fields is necessary in order to obtain an overall agreement with the experimental time evolution during the transformation process (both microstructure and transformed fraction), and provides a natural mechanism to stop the primary crystallization. However, the main picture of the final microstructure developed is rather insensitive to the overlapping diffusion field consideration.

The validity of the model has been tested against experimental data on the primary crystallization of an amorphous material resulting in a nanocrystalline structure. In practical applications, interesting macroscopical properties of new materials may be obtained with a very large amount of grains of a refined nanostructure, namely, a very small grain size distribution with a small average grain size. The results presented show that those characteristics may be achieved as a result of a diffusion-controlled growth mechanism.

\section{ACKNOWLEDGMENTS}

This work was financed by CICYT, Grant Nos. PB941209 and MAT96-0692, UPC, Grant No. PR9505, and Generalitat de Catalunya, Grant No. 1995SGR 00514. 
*Electronic address: crespo@benard.fa.upc.es

${ }^{1}$ W. Kurtz and D. J. Fisher, Fundamentals of Solidification, 3rd. ed. (Trans Tech Publications, Aedermannsdorf, Switzerland, 1992).

${ }^{2}$ A. N. Kolmogorov, Bull. Acad. Sci. USSR, Phys. Ser. 1, 355 (1937).

${ }^{3}$ W. A. Johnson and P. A. Mehl, Trans. Am. Inst. Min. Metall. Eng. 135, 416 (1939).

${ }^{4}$ M. Avrami, J. Chem. Phys. 7, 1103 (1939).

${ }^{5}$ M. Avrami, J. Chem. Phys. 8, 212 (1940).

${ }^{6}$ M. Avrami, J. Chem. Phys. 9, 177 (1941).

${ }^{7}$ J. W. Cahn, in Thermodynamics and Kinetics of Phase Transformations, edited by J. S. Im, B. Park, A. L. Green, and G. B. Stephenson, MRS Symposia Proceedings No. 398 (Materials Research Society, Pittsburgh, (1996).

${ }^{8}$ D. R. Uhlmann, J. Non-Cryst. Solids 7, 337 (1972).

${ }^{9}$ M. C. Weinberg, J. Non-Cryst. Solids 142, 126 (1992).

${ }^{10}$ N. Clavaguera, J. Non-Cryst. Solids 162, 40 (1993).

${ }^{11}$ N. Clavaguera and M. T. Clavaguera-Mora, Mater. Sci. Eng. A 179, 288 (1994).

${ }^{12}$ D. Crespo and T. Pradell, Phys. Rev. B 54, 3101 (1996).

${ }^{13} \mathrm{We}$ use this simplified model to account for any transient nucleation and Gibbs-Thomson effects at initial stages of grain growth.

${ }^{14}$ Y. Yoshizawa, S. Oguma, and K. Yamauchi, J. Appl. Phys. 64, 6044 (1988).

${ }^{15}$ Y. Yoshizawa and K. Yamauchi, Mater. Trans. JIM 31, 307 (1990).

${ }^{16}$ T. H. Noth, M. B. Lee, H. J. Kim, and I. K. Kang J. Appl. Phys. 67, 5568 (1990).
${ }^{17}$ K. Hono, K. Hiraga, Q. Wang, A. Inoue, and T. Sakurai, Surf. Sci. 266, 385 (1992).

${ }^{18}$ G. Herzer, Phys. Scr. T49, 307 (1993).

${ }^{19}$ A. R. Yavari and O. Drbohlav, Mater. Trans. JIM 36, 896 (1995).

${ }^{20}$ D. Turnbull, Contemp. Phys. 10, 473 (1969).

${ }^{21}$ N. Clavaguera, T. Pradell, J. Zhu, and M. T. Clavaguera-Mora, Nanostruct. Mater. 6, 453 (1995).

${ }^{22}$ J. Zhu, Ph.D. thesis, Universitat Autònoma de Barcelona, 1996.

${ }^{23} \mathrm{~J}$. W. Christian, The Theory of Transformations in Metals and Alloys (Pergamon Press, Oxford, 1975).

${ }^{24}$ C. Wert and C. Zener, J. Appl. Phys. 20, 962 (1949).

${ }^{25}$ T. Pradell, N. Clavaguera, J. Zhu, and M. T. Clavaguera-Mora, J. Phys. Condens. Matter 7, 4129 (1995).

${ }^{26}$ T. Pradell, N. Clavaguera, J. Zhu, and M. T. Clavaguera-Mora, in ICAME '95, Conference Proceedings of the Societa Italiana di Fisica, edited by I. Ortalli (Societa Italiana di Fisica, Bologna, 1996), Vol. 50, p. 409.

${ }^{27}$ H. Tomita and S. Miyashita, Phys. Rev. B 46, 8886 (1992).

${ }^{28}$ P. A. Rikvold, H. Tomita, S. Miyashita, and S. W. Sides, Phys. Rev. E 49, 5080 (1994).

${ }^{29}$ P. D. Beale, Integrat. Ferroelectr. 4, 107 (1994).

${ }^{30}$ G. Herzer, IEEE Trans. Magn. MAG-26, 1397 (1990).

${ }^{31}$ N. Clavaguera and M. T. Clavaguera-Mora, in Thermodynamics and Kinetics of Phase Transformations, edited by J. S. Im, B. Park, A. L. Greer, and G. B. Stephenson, MRS Symposia Proceedings No. 398 (Materials Research Society, Pittsburgh, 1996), p. 319.

${ }^{32}$ J. Kohlbrecher, A. Wiedenmann, and H. Wollenberger, Physica B 213\&214, 579 (1995).

${ }^{33}$ S. A. Saltykov, Stereology (Springer-Verlag, New York, 1967), p. 163. 